\title{
Protection of Trigonelline on Experimental Diabetic Peripheral Neuropathy
}

\author{
Ji-Yin Zhou and Shi-Wen Zhou \\ Base for Drug Clinical Trial, Xinqiao Hospital, Third Military Medical University, Chongqing 400037, China \\ Correspondence should be addressed to Shi-Wen Zhou, zhoushiwen2007@yahoo.com
}

Received 17 July 2012; Accepted 4 November 2012

Academic Editor: Bashar Saad

Copyright ( $) 2012$ J.-Y. Zhou and S.-W. Zhou. This is an open access article distributed under the Creative Commons Attribution License, which permits unrestricted use, distribution, and reproduction in any medium, provided the original work is properly cited.

\begin{abstract}
The mechanisms leading to diabetic peripheral neuropathy are complex and there is no effective drug to treat it. As an active component of several traditional Chinese medicines, trigonelline has beneficial effects on diabetes with hyperlipidemia. The protective effects and the mechanism of trigonelline on diabetic peripheral neuropathy were evaluated in streptozotocin- and highcarbohydrate/high-fat diet-induced diabetic rats. Rats were divided into four groups at the end of week 2: control, diabetes, diabetes + trigonelline $(40 \mathrm{mg} / \mathrm{kg})$, and diabetes + sitagliptin $(4 \mathrm{mg} / \mathrm{kg})$. After 48 -week treatment, technologies of nerve conduction, cold and hot immersion test, transmission electron microscopy, real-time PCR, and Western blotting were applied. Serum glucose, serum insulin, insulin sensitivity index, lipid parameters, body weight, sciatic nerve conduction velocity, nociception, glucagonlike peptide-1 receptor mRNA and protein, total and phosphorylated p38 mitogen-activated protein kinases protein expression, malonaldehyde content, and superoxide dismutase activity were altered in diabetic rats, and were near control levels treated with trigonelline. Slight micropathological changes existed in sciatic nerve of trigonelline-treated diabetic rats. These findings suggest that trigonelline has beneficial effects for diabetic peripheral neuropathy through glucagon-like peptide-1 receptor/p38 mitogenactivated protein kinases signaling pathway, nerve conduction velocity, antioxidant enzyme activity, improving micropathological changes of sciatic nerve and decreasing lipid peroxidation.
\end{abstract}

\section{Introduction}

As a common complication of diabetes, diabetic peripheral neuropathy (DPN) affects likely up to one-third of adults with diabetes [1]. The most common form of DPN is a sensory polyneuropathy with symptoms such as paresthesia, unremitting pain (hyperalgesia), and reduced temperature and vibration perception thresholds. Clinical trials have failed to demonstrate the effectiveness of any drug treatment in stabilizing or improving nerve function, and only symptomatic pain therapies with variable efficacy are available [2]. First and foremost, treatment of DPN centers on control of the patient's blood glucose level. Nowadays, the only agents labeled for the treatment of DPN include lidocaine patches $5 \%$, duloxetine, gabapentin, and pregabalin. Despite clinical use of these agents, the successful therapy of DPN remains a challenge [3]. Since pathogenic complexity of DPN, new therapeutic interventions that target primary mechanisms contributing to nerve damage are critical for the future treatment of this complication.

Recently, diabetic healthcare professions had a considerable interest in regarding complementary and alternative approaches, including by identifying natural neuroprotective constituent from herb medicine to replace synthetic ones $[4,5]$. As we recently reviewed [6], trigonelline, a major ingredient of several traditional Chinese medicine with anti-diabetic effect $[7,8]$, has a hypoglycemic effect both in rats [9-12] and humans [13, 14] and has antioxidant effectiveness in vitro $[15,16]$. Recently the study in our laboratory showed that trigonelline had a beneficial effect for diabetes through decreasing blood glucose and lipid levels, increasing insulin sensitivity index and insulin content, upregulating antioxidant enzyme activity, and decreasing lipid peroxidation [17]. Trigonelline also is one of the main components of Mirabilis jalapa L. root which possesses 
both potential insulin sensitivity and hypoglycemic and hypolipidemic effects on diabetes [18].

Endogenous glucagon-like peptide-1 (GLP-1) is an insulinotropic peptide synthesized and secreted from the Lcells of the gastrointestinal tract in response to food intake. When given exogenously, GLP-1 improves glucostasis in type 2 diabetic patients, primarily by stimulating endogenous insulin secretion. GLP-1 and related peptides possess neural functions including neurotrophic and neuroprotective effects, in addition to glucoregulatory and energy balance functions [19]. Dipeptidyl peptidase IV inhibitor inhibits degradation of GLP-1 by the dipeptidyl peptidase IV enzyme. Several researches show that GLP-1 and dipeptidyl peptidase IV inhibitor, such as Exendin-4 and vildagliptin, offer protection against the experimental DPN [20-24]. GLP-1 receptor stimulation preserves primary cortical and dopaminergic neurons in cellular and rodent models of stroke and Parkinsonism [25]. Peripheral nerve of diabetic rodents had functional GLP-1R and GLP-1R-mediated ERKsignaling in sciatic nerve of diabetic rodents protecting large motor fibre function and small C fibre structure by a mechanism independent of glycaemic control [26]. Diabetes is known to activate p38 MAPK in nerve tissues in both rats [27] and patients and inhibition of p38 MAPK has beneficial effects related to nerve function [28]. But the function of GLP-1R-mediated p38 mitogen-activated protein kinases (MAPK) signaling pathway in the DPN is still unknown.

With the above background, the present study systematically investigated the beneficial effect of trigonelline on peripheral neuropathy in low-dose streptozotocin and high-carbohydrate/high-fat diet-induced diabetic rats, by technologies of nerve conduction, cold and hot immersion test, transmission electron microscopy, real-time PCR, and Western blotting. The efficacy was compared with a standard hypoglycemic drug, dipeptidyl peptidase IV inhibitor, and sitagliptin.

\section{Materials and Methods}

2.1. Experimental Animals. Male Wistar rats, weighing 180 $220 \mathrm{~g}$, were purchased from Research Institute of Surgery Experimental Animal Center and bred in specific pathogenfree condition. All experiments were performed with the approval of the Animal Studies Ethics Committee of Xinqiao Hospital, Third Military Medical University. All animals were separately housed in cages (one rat per cage). Rats were allowed to eat a standard diet and drink ad libitum and adapted to the experimental conditions at $20 \pm 2^{\circ} \mathrm{C}$, humidity $60 \pm 5 \%$ with a fixed $12 \mathrm{~h}$ artificial light period for one week.

2.2. Chemicals and Reagents. Trigonelline hydrochloride (trigonelline, purity 100\%) was brought from Sigma-Aldrich Trading Co., Ltd, USA. Sitagliptin phosphate (sitagliptin, Januvia) was provided by Merck Pharmaceuticals, WhiteHouse Station, NJ, USA. Streptozotocin and $\beta$-actin polyclonal antibody were brought from Sigma Chemicals, St. Louis, MO, USA. Rabbit polyclonal antibody to GLP-1R and total and phosphorylated p38 MAPK were brought from Santa Cruz, USA. Insulin radioimmunoassay kit was provided by Beijing North Institute of Biological Technology, China. The kits of hemoglobin $\mathrm{A}_{1 \mathrm{c}}\left(\mathrm{HbA}_{1 \mathrm{c}}\right)$, total cholesterol, triglyceride, superoxide dismutase (SOD), malonaldehyde, and protein assay were obtained from Nanjing Jiancheng Bioengineering Institute, China. Other chemicals were of analytical reagent grade from commercial sources.

\subsection{Induction of Diabetes and Drug Treatment. Four-treat-} ment groups were defined: control, diabetes, diabetes plus trigonelline, and diabetes plus sitagliptin. Diabetes was induced by low-dose streptozotocin treatment $(35 \mathrm{mg} / \mathrm{kg}$, i.p.) and high-carbohydrate/high-fat diet (70\% standard diet, $12 \%$ lard, $9 \%$ yolk powder, and $9 \%$ plantation white sugar) [29]. The animals with fasting blood glucose level of above $16.7 \mathrm{mmol} / \mathrm{L}$ were used as diabetic ones two weeks after streptozotocin injection. Trigonelline $(40 \mathrm{mg} / \mathrm{kg})$ and sitagliptin $(4 \mathrm{mg} / \mathrm{kg})$ were mixed daily with a vehicle consisting of the standard diet to diabetic animals for 48 weeks. The standard diet or the high-carbohydrate/highfat diet was given only after the vehicle was completely ingested by the animals. There were 10 rats in each group. Animal weight was measured every 2 weeks throughout the experiment and the drug dose was accordingly adjusted. Fasting blood glucose levels were measured every 8 weeks to document the persistence of diabetes. After an $8 \mathrm{~h}$ fast, one drop of tail blood was analyzed using a standard Glucometer (OneTouch; LifeScan Inc., Milpitas, CA).

2.4. Cold Immersion Test and Hot Immersion Test. The animals were subjected to cold immersion test $\left(10^{\circ} \mathrm{C}\right)$ and hot immersion test $\left(45^{\circ} \mathrm{C}\right)$, which is the immersion of the rat tail in water maintained at the mentioned temperature and then tail flick latency or signs of struggle was observed. The cut off time was $15 \mathrm{~s}$ [30].

2.5. Nerve Conduction Studies. Nerve conduction velocity (NCV) was recorded as previously described [31, 32]. For the sciatic nerve, the recording electrodes (AD Instruments, PowerLab/16SP) were placed in the dorsum of the foot, and the stimulating electrodes at the knee and sciatic notch. For the sural nerve, the anode was placed on the third toe of the foot, and the cathode was placed on the heel of the foot. The cathode and anode were placed $5 \mathrm{~mm}$ apart. The frequency band was inclusive of two $10 \mathrm{~Hz}$ muscle potential recordings (orthodromic, motor) and $102 \mathrm{~Hz}$ potential recordings (antidromic, sensory).

2.6. Tissue Harvest. Forty-eight weeks after drug treatment, rats were anaesthetized by i.p. sodium pentobarbital $(60 \mathrm{mg} / \mathrm{kg})$ and opened the abdominal cavity. Cannula was inserted in portal vein and portal blood samples were collected in tubes containing dipeptidyl peptidase IV inhibitor $(10 \mathrm{~mL} / \mathrm{mL}$, ADL Research, Santa Barbara, CA, USA) via the portal vein, and a blood sample $(50 \mu \mathrm{L})$ was used for measurement of $\mathrm{HbA}_{1 \mathrm{c}}$. Blood samples were allowed to clot to get the serum which was stored at $-70^{\circ} \mathrm{C}$ for assessment of GLP-1 (7-36) amide and other biochemical parameters. 
Rats were then killed immediately and tissues were harvested as previously described [33, 34]. The left sciatic nerve was dissected and rapidly frozen by immersion in liquid nitrogen for gene and protein expression analysis. The right sciatic nerve was dissected, and parts of it were immediately submerged in ice-cold antioxidant buffer, rapidly frozen by immersion in liquid nitrogen, and stored at $-70^{\circ} \mathrm{C}$ for quantification; other parts were fixed for transmission electron microscope.

2.7. Gene Expression. Total RNA from frozen tissue was extracted using the RNA-out kit. Complementary DNA was synthesized with a cDNA synthesis kit. The real-time PCR measurement of individual cDNA was performed using SYBR green dye to measure duplex DNA formation with the ABI Prism 7500 Sequence Detection System (Applied Biosystems, Foster City, CA), normalized to the amount of $\beta$-actin RNA, and analyzed by the $2^{-\triangle \Delta C T}$ method [35]. The specific PCR primers of GLP-1R were used: $5^{\prime}$-TGA ACC TGT TTG CAT CCT TCA- $3^{\prime}$ and $5^{\prime}$-ACT TGG CAA GCC TGC ATT TGA-3' (Accession No. NM_021332) as described [36].

2.8. Protein Expression by Western Blot. Western blot analysis was carried out as we previously reported [37]. For each experiment samples were run in duplicates. The densitometry analysis of the image was performed by Image-Pro Plus 6.0 (Media Cybernetics, Silver Spring, USA).

2.9. Plasma Biochemical Parameters Measurement. HbA $1 \mathrm{c}$, serum total cholesterol (TC), and triglyceride (TG) were measured by HITACHI 7170 automatic biochemistry analyzer (HITACHI, Japan) using commercial kit, respectively. For GLP-1 contents determination, blood was drawn into heparinized tubes containing EDTA and dipeptidyl peptidase IV inhibitor (inhibits degradation of GLP-1 by the dipeptidyl peptidase IV enzyme present in serum) by radioimmunoassay (Beijing gersion Bio-Technology Co., Ltd, China) [20]. SOD activity was assayed by the method of Kakkar et al. [38]. Malonaldehyde content was estimated by the method of Draper and Hadley [39]. Serum insulin concentration was measured by commercial kits according to the manufacturer's instructions. Insulin sensitivity index $=\log (1 /$ fasting plasma glucose $\times$ serum insulin).

2.10. Micropathological Measurement. For transmission electron microscopy, 3-4 mm lengths of sciatic nerve of different groups of rats were obtained and fixed overnight in $2 \%$ glutaraldehyde ( $\mathrm{pH} 7.3$ ) in $0.1 \mathrm{~mol} / \mathrm{L}$ phosphate buffer saline at $4{ }^{\circ} \mathrm{C}$. The specimens were then fixed in $2 \%$ osmium tetroxide $(0.1 \mathrm{~mol} / \mathrm{L})$, dehydrated through a graded series of acetone, and embedded in araldite. One-micron sections were cut and then stained with toluidine blue. Suitable areas for ultrastructural study were chosen after examining $1 \mu$ sections (70-80 nm), were cut on an LKB-5 ultramicrotome (Sweden) using a diamond knife and sections were mounted on a copper grid and stained with uranyl acetate and Reynolds lead citrate [40]. They were examined and photographed using a transmission electron microscope (Hitachi-7500, made by Hitachi, Japan).

2.11. Statistical Analysis. Data were expressed as mean \pm SD. All the grouped data were statistically performed with SPSS 13.0. Significant differences between means were evaluated by one-way analysis of variance (ANOVA), followed by multiple comparisons with least significant difference (LSD) test or Tukey's test where appropriate. $P<0.05$ was considered to indicate statistical significance.

\section{Results}

3.1. Effects of Drugs on Serum Glucose, Insulin, Lipid Parameters, and Body Weight. Before drug administration (week 0), diabetic rats had high baseline fasting blood glucose levels. Blood glucose level of diabetic rats increased significantly comparing to that of the control ones. During drug treatment, trigonelline gradually decreased fasting blood glucose to normal level (Figure 1). $\mathrm{HbA}_{1 \mathrm{c}}$ level of diabetic rats was significantly higher than that of the control ones. Treatment with trigonelline and sitagliptin for 48 weeks reverted the increased diabetic $\mathrm{HbA}_{1 \mathrm{c}}$ level to near the control ones. Diabetic rats had significant increased-serum insulin concentration and decreased-insulin sensitivity index when compared with control ones. Trigonelline treatment reversed serum insulin level and insulin sensitivity index, but did not for sitagliptin. The TC and TG levels of diabetic rats were significantly higher than those of the control ones. Treatment with trigonelline for 48 weeks significantly decreased TC and TG levels, but sitagliptin did not affect these lipid metabolic parameters. Control rats grew faster than the other group ones and body weight of diabetic rats continued to increase (data not shown). The weight gain between initial (week 0) and final (week 48) body weight of control rats were remarkably higher than that of the other group ones. At weeks 48, trigonelline, but not sitagliptin, treatment obviously reduced diabetic weight gain (Table 1).

3.2. Effects of Drugs on NCV and Nociception. In 48th week postdiabetes, diabetic rats showed significant decrease in both motor and sensory NCV as compared to the age-matched control rats. Treatment with trigonelline and sitagliptin for 48 weeks significantly reversed the motor and sensory nerve conduction impairment in diabetic rats (Table 2).

Tail flick latency in 48th week was significantly decreased in diabetic animals in both cold as well as hot immersion test as compared to control animals. This decrease in tail flick latency was reversed significantly in treatment with trigonelline and sitagliptin (Table 2).

3.3. Electron Microscopic Analysis of Sciatic Nerve. Ultrastructural examination showed that sciatic nerve of control rats has normal micropathological morphology with integrity structural of nerve fibers and myelin, concentric circle-like arrangement of lamellar myelin, uniform electron density within axons, and normal structure of Schwann cells, 
TABLE 1: Effects of trigonelline on serum lipid parameters and body weight gain in diabetic rats.

\begin{tabular}{|c|c|c|c|c|c|c|}
\hline Group & HbAlc $(\%)$ & $\begin{array}{l}\text { Serum insulin } \\
(\mathrm{mU} / \mathrm{L})\end{array}$ & $\begin{array}{c}\text { Insulin } \\
\text { sensitivity index } \\
\end{array}$ & $\begin{array}{l}\text { Triglyceride } \\
(\mathrm{mmol} / \mathrm{L})\end{array}$ & $\begin{array}{l}\text { Total cholesterol } \\
(\mathrm{mmol} / \mathrm{L})\end{array}$ & $\begin{array}{l}\text { Weight gain } \\
(\mathrm{g})\end{array}$ \\
\hline Control & $37.41 \pm 8.53$ & $33.24 \pm 7.59$ & $-2.31 \pm 0.11$ & $1.76 \pm 0.24$ & $0.76 \pm 0.13$ & $253.7 \pm 18.0$ \\
\hline Diabetes & $59.44 \pm 10.12^{*}$ & $47.16 \pm 7.60^{*}$ & $-3.09 \pm 0.06^{*}$ & $2.42 \pm 0.46^{*}$ & $2.14 \pm 0.18^{*}$ & $137.3 \pm 7.6^{*}$ \\
\hline Diabetes + trigonelline & $39.50 \pm 10.75^{\# \#}$ & $36.80 \pm 8.13^{\# \#}$ & $-2.38 \pm 0.12^{\# \#}$ & $1.85 \pm 0.21^{\# \#}$ & $0.79 \pm 0.13^{\# \#}$ & $103.0 \pm 4.3^{\# \#}$ \\
\hline Diabetes + sitagliptin & $37.69 \pm 7.81^{\# \#}$ & $45.79 \pm 8.01$ & $-2.46 \pm 0.11$ & $2.56 \pm 0.51$ & $2.18 \pm 0.24$ & $139.2 \pm 12.3^{\#}$ \\
\hline
\end{tabular}

Data are given as mean $\pm \mathrm{SD}, n=10 .{ }^{*} P<0.01$, compared with control rats; ${ }^{\#} P<0.05,{ }^{\# \#} P<0.01$, compared with diabetes rats.

TABLE 2: Effects of trigonelline on motor and sensory nerve conduction velocity and tail flick latency in diabetic rats.

\begin{tabular}{lcccc}
\hline Group & $\begin{array}{c}\text { Motor nerve conduction } \\
\text { velocity }(\mathrm{m} / \mathrm{s})\end{array}$ & $\begin{array}{c}\text { Sensory nerve conduction } \\
\text { velocity }(\mathrm{m} / \mathrm{s})\end{array}$ & $\begin{array}{c}\text { Tail flick latency in cold } \\
\text { immersion }(\mathrm{s})\end{array}$ & $\begin{array}{c}\text { Tail flick latency in hot } \\
\text { immersion }(\mathrm{s})\end{array}$ \\
\hline Control & $55.34 \pm 5.92$ & $57.61 \pm 6.85$ & $14.5 \pm 1.0$ & $14.2 \pm 0.9$ \\
Diabetes & $35.04 \pm 4.36^{*}$ & $40.17 \pm 4.03^{*}$ & $5.2 \pm 1.0^{*}$ & $6.3 \pm 0.7^{*}$ \\
Diabetes + trigonelline & $49.74 \pm 5.05^{\# \#}$ & $51.76 \pm 4.82^{\# \#}$ & $12.4 \pm 1.1^{\# \#}$ & $12.0 \pm 1.3^{\# \#}$ \\
Diabetes + sitagliptin & $41.05 \pm 5.27^{\#}$ & $47.68 \pm 3.38^{\# \#}$ & $10.8 \pm 0.8^{\# \#}$ & $10.7 \pm 1.0^{\# \#}$ \\
\hline
\end{tabular}

Data are given as mean $\pm \mathrm{SD}, n=10 .{ }^{*} P<0.01$, compared with control rats; ${ }^{\#} P<0.05$, ${ }^{\#} P<0.01$, compared with diabetes rats.

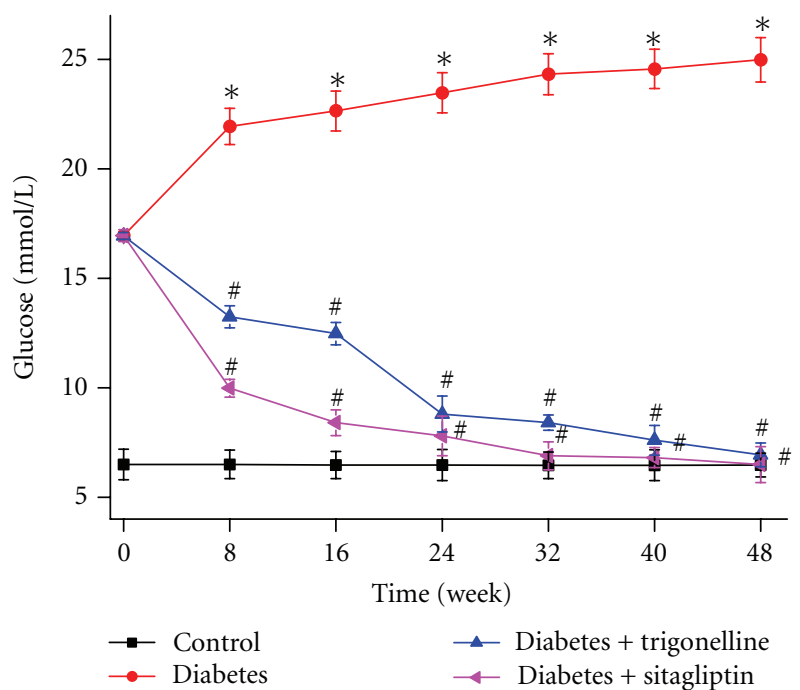

Figure 1: Effect of trigonelline on glucose of diabetic rats. Data are given as mean $\pm \mathrm{SD}(n=10) .{ }^{*} P<0.01$ versus control rats; ${ }^{\#} P<$ 0.01 versus diabetes rats.

mitochondria, and unmyelinated fibers (Figure 2(C)). But diabetic rats showed significant separation of myelinated nerve fiber myelin lamellar, lost layered-structure, disordered arrangement, faded electron density, thinner axons, neurofilament disorder, and swollen mitochondria of Schwann cells (Figure 2(D)). The protective effects of trigonelline were evident with ameliorated micropathology of diabetic sciatic nerve (Figure 2(T)), the same as sitagliptin (Figure 2(S)).

3.4. Effects of Drugs on GLP-1 Level in Serum and GLP$1 R$ Expression in Sciatic Nerve. GLP-1 level in serum and GLP-1R mRNA and protein expression in sciatic nerve were all significantly decreased in diabetic rats. Forty-eightweek treatment with trigonelline and sitagliptin significantly increased GLP-1 level in diabetic serum to near that of control rats. Trigonelline and sitagliptin significantly increased the downregulated GLP-1R mRNA and protein expression in diabetic sciatic nerve to near those of control rats (Table 3 and Figures 3(a) and 3(b)).

3.5. Effects of Drugs on Total and Phosphorylated $p 38$ MAPK Protein Expression in Sciatic Nerve. Phosphorylated p38 MAPK protein expression in sciatic nerve significantly increased, whereas total p38 MAPK expression was unchanged in diabetic rats compared with nondiabetic control ones. Forty-eight-week treatment with trigonelline and sitagliptin significantly decreased the upregulated phosphorylated p38 MAPK protein expression in diabetic sciatic nerve to near those of control rats and still did not affect total phosphorylated p38 MAPK protein expression (Figures 3(a), $3(\mathrm{c})$, and $3(\mathrm{~d}))$.

3.6. Effects of Drugs on Superoxide Dismutase Activity and Malonaldehyde Content in Serum. Changes in the activities of superoxide dismutase and malonaldehyde in rat serum are depicted in Table 4. Significantly decreased superoxide dismutase activity and elevated malonaldehyde content were observed in serum of diabetic rats. Significant raise in the reduced superoxide dismutase activity and significant reduction in the increased malonaldehyde content were observed in diabetic rat and reverted back to near control levels after 48-week treatment with trigonelline and sitagliptin.

\section{Discussion}

DPN is a common complication of type 1 and 2 diabetes, resulting from hyperglycemia-induced oxidative stress [41]. The current study evaluated the effects of trigonelline on the development of streptozotocin-induced DPN rats. We found that: (1) trigonelline significantly improved motor and sensory NCV and nociception; (2) trigonelline has protection on the neuron tissue (sciatic nerve); (3) trigonelline reduced oxidative stress in the sciatic nerve; and (4) GLP-1R and p38 


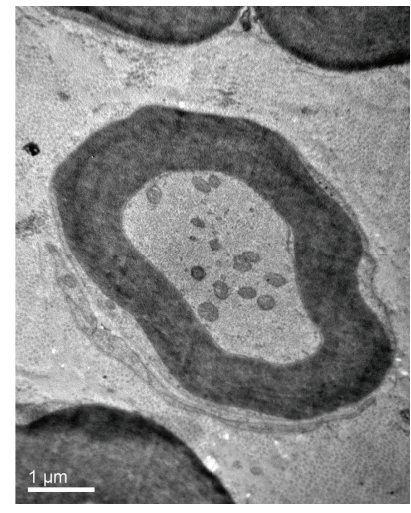

C

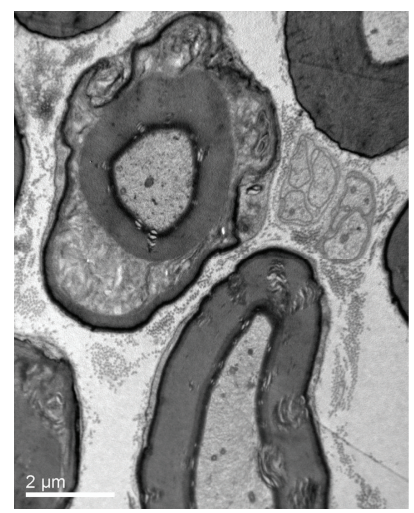

$\mathrm{D}$

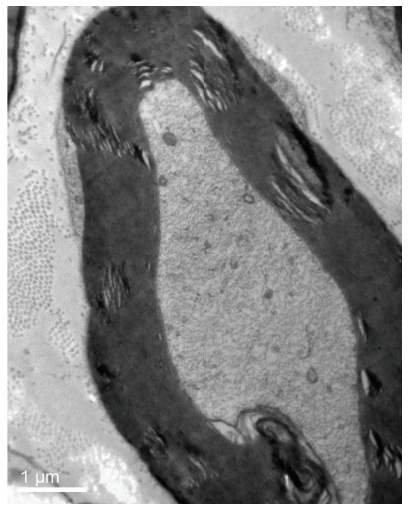

$\mathrm{T}$

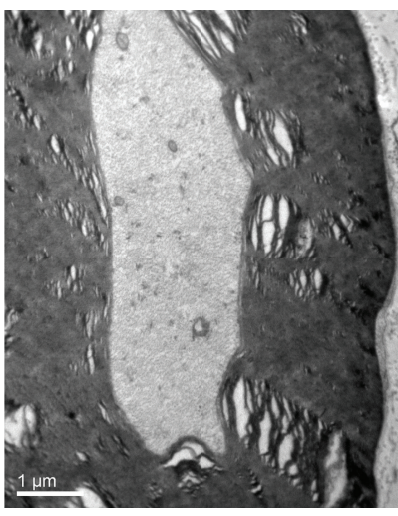

S

FIGURE 2: Effects of trigonelline on the micromorphology of sciatic nerve. C, control rats; $\mathrm{D}$, diabetic rats; T, trigonelline-treated diabetes; $\mathrm{S}$ sitagliptin-treated diabetes.

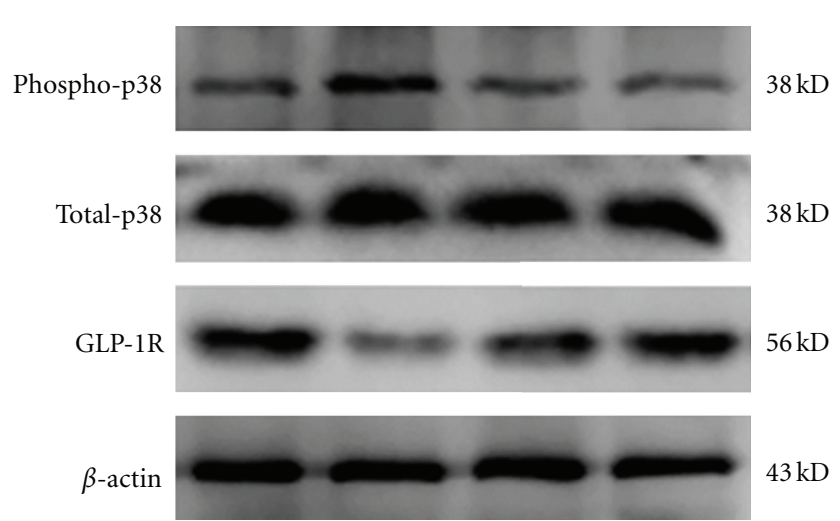

(a)

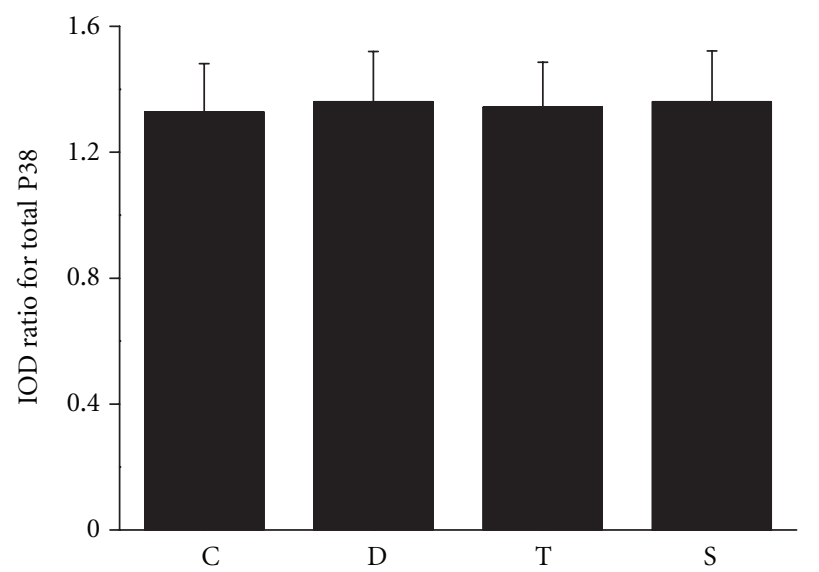

(c)

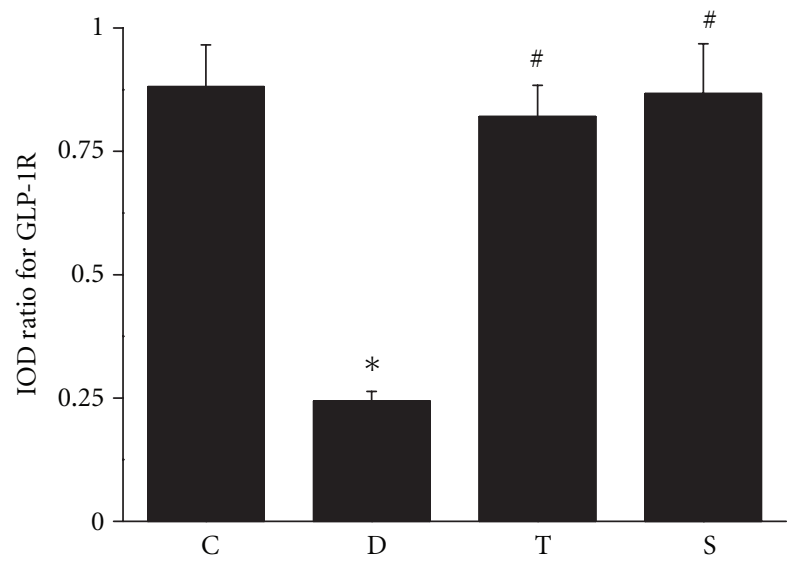

(b)

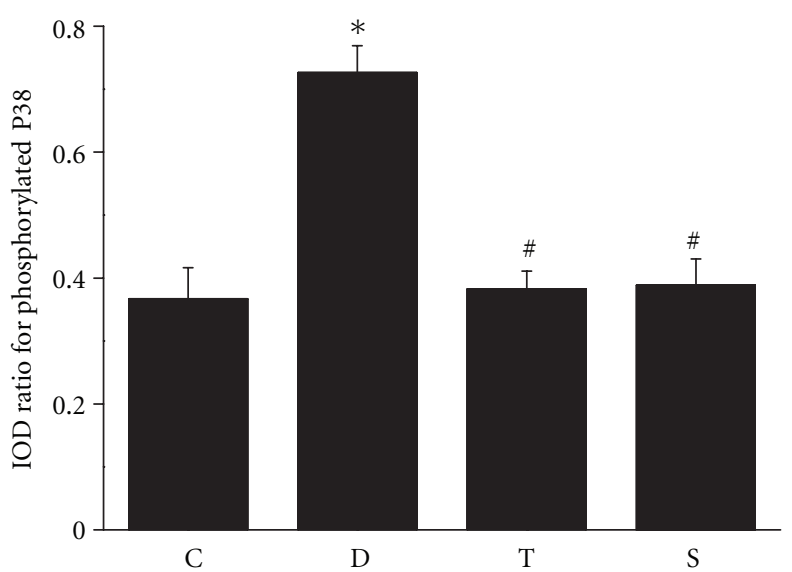

(d)

FIGURE 3: Effects of trigonelline on GLP-1R protein expression in sciatic nerve. Densitometric analysis of the bands is expressed as integrated optical density (IOD), corrected for the corresponding $\beta$-actin. C, control rats; $\mathrm{D}$, diabetic rats; T, trigonelline-treated diabetes; $\mathrm{S}$ sitagliptintreated diabetes. Data are given as mean $\pm \mathrm{SD}(n=10) .{ }^{*} P<0.01$ versus control rats; ${ }^{\#} P<0.01$ versus diabetes rats. 
TABLE 3: Effects of trigonelline on GLP-1 level in serum and GLP-1R mRNA expression in sciatic nerve.

\begin{tabular}{lcr}
\hline Group & GLP-1 level in serum $(\mathrm{pmol} / \mathrm{L})$ & Sciatic nerve GLP-1R mRNA \\
\hline Control & $19.06 \pm 2.65$ & $1.00 \pm 0.00$ \\
Diabetes & $12.42 \pm 1.11^{*}$ & $0.49 \pm 0.08^{*}$ \\
Diabetes + trigonelline & $18.90 \pm 1.25^{\#}$ & $1.03 \pm 0.12^{\#}$ \\
Diabetes + sitagliptin & $18.27 \pm 1.22^{\#}$ & $1.08 \pm 0.12^{\#}$ \\
\hline
\end{tabular}

$\Delta \mathrm{CT}$ (threshold cycle) $=\mathrm{CT}$ (target gene) $-\mathrm{CT}\left(\beta\right.$-actin), $\Delta \Delta \mathrm{CT}=\Delta \mathrm{CT}$ (other rats) $-\Delta \mathrm{CT}$ (control rats), relative fold $=2^{-\Delta \Delta \mathrm{CT}}$, and control rat is 1 . Data are given as mean $\pm \mathrm{SD}, n=10$. ${ }^{*} P<0.01$, compared with control rats; ${ }^{*} P<0.01$, compared with diabetes rats.

TABLE 4: Effects of trigonelline on SOD activity and malonaldehyde content in serum.

\begin{tabular}{lcc}
\hline Group & SOD $(\mathrm{U} / \mathrm{mL})$ & $\begin{array}{c}\text { Malonaldehyde } \\
(\mathrm{nmol} / \mathrm{mL})\end{array}$ \\
\hline Control & $124.04 \pm 4.46$ & $4.19 \pm 0.78$ \\
Diabetes & $83.94 \pm 3.31^{*}$ & $6.07 \pm 0.97^{*}$ \\
Diabetes + trigonelline & $115.30 \pm 7.87^{\#}$ & $4.57 \pm 0.95^{\#}$ \\
Diabetes + sitagliptin & $97.99 \pm 4.95$ & $5.91 \pm 1.41$ \\
\hline
\end{tabular}

Data are given as mean $\pm \mathrm{SD}, n=10$. ${ }^{*} P<0.01$, compared with control rats; ${ }^{\#} P<0.01$, compared with diabetes rats.

MAPK activity were regulated by trigonelline and are likely relevant to the development of DPN.

The streptozotocin-induced diabetic rat is an established model of type 2 diabetes that develops a profound neuropathy [42]. Using a low-dose streptozotocin and a high-carbohydrate/high-fat diet protocol, we confirmed diabetes induction and animal survival for 48 weeks. The streptozotocin-induced diabetic rats developed increased fasting blood glucose, and $\mathrm{HbA}_{1 c}$ and gained weight relative to control rats, consistent with previous reports [29]. Forty-eight-week treatment with trigonelline had antihyperlipidemic effects and lowered blood glucose, $\mathrm{HbA}_{1 \mathrm{c}}$, and body weight on DPN rats. As dyslipidemia is a significant contributor to the DPN development, hypolipidemic agent may prevent or reverse diabetes-induced nerve degeneration [43].

We demonstrated the effects of trigonelline on GLP1R/p38 MAPK signaling pathway in streptozotocin-induced diabetic mice. Forty-eight-week treatment with trigonelline increased serum GLP-1 level and GLP-1R expression and decreased the upregulated phosphorylated p38 MAPK activity, but did not affect total p38 MAPK protein expression in the sciatic nerve. Although the precise mechanisms still unclear, the effects of trigonelline on DPN may be partly attributed to its regulation on GLP-1R/p38 MAPK signaling pathway. Exogenous GLP-1R activation significantly reduces glucose-dependent reactive oxygen species generation in hypothalamus [44]. The antioxidative effects of GLP-1R agonists, such as exendin-4, have regenerative effects on peripheral nervous systems. In the dorsal root ganglia of streptozotocin-induced diabetic rats, p38 were activated by oxidative-nitrosative stress [45].

Streptozotocin-induced diabetic rats developed persistent DPN after 48-week diabetes as measured by both increased thermal latency and decreased NCV. Thermal latency was significantly reduced by trigonelline treatment, and NCV studies demonstrated a corresponding trend toward improvement. We also observed the separation of myelinated-nerve fiber myelin lamellar, lost layeredstructure, disordered arrangement, faded electron density, thinner axons, neurofilament disorder, and swollen mitochondria of Schwann cells of sciatic nerve by transmission electron microscopy. These ultrastructure changes were improved by 48 -week treatment with trigonelline. Several studies showed that trigonelline could increase the excitability of dorsal root ganglion neurons [46], promote functional neurite outgrowth [47], and prevent both dendritic and axonal atrophy induced by amyloid $\beta(25-35)$ in a dosedependent manner [48]. The histological studies performed 50 weeks after diabetic induction suggest that the neuroprotection provided by trigonelline leads to long-lasting preservation of sciatic nerve and its function.

Trigonelline treatment had a hypoglycemic effect and partially corrected DPN. Trigonelline has an antioxidant effectiveness in cell-free systems, human colon cell lines [15], and on liposome peroxidation [16]. To determine whether the amelioration of DPN observed is due to a reduction of diabetes-induced oxidative stress, biomarkers of oxidative damage were assayed in serum. Serum SOD activity was significantly lowered, and malonaldehyde content was significantly increased in the streptozotocin-induced diabetic rats, consistent with previous work showing that diabetes increases oxidized lipids $[49,50]$. Our findings are consistent with the oxidative stress model of DPN [41] because thermal latency is highly correlated with increased level of malonaldehyde and decreased level of SOD. Treatment with trigonelline reduced both biomarkers significantly, to levels near that of control animals. Because trigonelline corrected both hyperglycemia and reduced oxidative stress, we concluded that trigonelline promoted antioxidant activity in DPN. Many drugs with antioxidant function are effective in treating animal models of DPN, including the antioxidant response element activator resveratrol [50] and innate antioxidant, genistein, and baicalein edaravone [49, 51, 52].

In conclusion, this study identifies trigonelline as a multitarget drug alleviating several manifestations of DPN. These results provide the rationale for screening animal experimental studies of alkaloids, especially those with improved pharmacological profile, such as oral administration, fewer adverse effects, and acceptable pharmaceutical features, as potential therapeutics for DPN and even for other diabetic complications. 


\section{Acknowledgments}

The authors gratefully acknowledge the funding from the National Natural Science Foundation of China (no. 81100597), Natural Science Foundation Project of Chongqing Science and Technology Commission (no. CSTC 2009BA5012), and the Natural Science Foundation of Third Military Medical University (no. 2009XQN34).

\section{References}

[1] M. S. Pinzur, "Diabetic Peripheral Neuropathy," Foot and Ankle Clinics, vol. 16, no. 2, pp. 345-349, 2011.

[2] A. J. M. Boulton, A. I. Vinik, J. C. Arezzo et al., "Diabetic neuropathies: a statement by the American Diabetes Association," Diabetes Care, vol. 28, no. 4, pp. 956-962, 2005.

[3] A. A. Habib and T. H. Brannagan III, "Therapeutic strategies for diabetic neuropathy," Current Neurology and Neuroscience Reports, vol. 10, no. 2, pp. 92-100, 2010.

[4] C. L. Chang and C. S. Lin, "Phytochemical composition, antioxidant activity, and neuroprotective effect of terminalia chebula retzius extracts," Evidence-Based Complementary and Alternative Medicine, vol. 2012, Article ID 125247, 7 pages, 2012.

[5] Y. F. Xian, Z. X. Lin, Q. Q. Mao et al., "Bioassay-guided isolation of neuroprotective compounds from Uncaria rhynchophylla against beta-amyloid-induced neurotoxicity," EvidenceBased Complementary and Alternative Medicine, vol. 2012, Article ID 802625, 8 pages, 2012.

[6] J. Zhou, L. Chan, and S. Zhou, “Trigonelline: a plant alkaloid with therapeutic potential for diabetes and central nervous system disease," Current Medicinal Chemistry, vol. 19, no. 21, pp. 3523-3531, 2012.

[7] T. Zia, S. N. Hasnain, and S. K. Hasan, "Evaluation of the oral hypoglycaemic effect of Trigonella foenum-graecum L. (methi) in normal mice," Journal of Ethnopharmacology, vol. 75, no. 2-3, pp. 191-195, 2001.

[8] S. Thakran, M. R. Siddiqui, and N. Z. Baquer, "Trigonella foenum graecum seed powder protects against histopathological abnormalities in tissues of diabetic rats," Molecular and Cellular Biochemistry, vol. 266, no. 1-2, pp. 151-159, 2004.

[9] R. Moorthy, K. Prabhu, and P. Murthy, "Studies on the isolation and effect of orally active hypoglycemic principle from the seeds of fenugreek (Trigonella foenum graecum)," Diabetes Bulletin, vol. 9, pp. 69-72, 1989.

[10] J. Mishkinsky, B. Joseph, and F. G. Sulman, "Hypoglycaemic effect of trigonelline,” The Lancet, vol. 2, no. 7529, pp. 13111312, 1967.

[11] O. Yoshinari and K. Igarashi, "Anti-diabetic effect of trigonelline and nicotinic acid, on KK-A ${ }^{y}$ mice," Current Medicinal Chemistry, vol. 17, no. 20, pp. 2196-2202, 2010.

[12] O. Yoshinari, H. Sato, and K. Igarashi, "Anti-diabetic effects of pumpkin and its components, trigonelline and nicotinic acid, on goto-kakizaki rats," Bioscience, Biotechnology and Biochemistry, vol. 73, no. 5, pp. 1033-1041, 2009.

[13] A. E. Van Dijk, M. R. Olthof, J. C. Meeuse, E. Seebus, R. J. Heine, and R. M. Van Dam, "Acute effects of decaffeinated coffee and the major coffee components chlorogenic acid and trigonelline on glucose tolerance," Diabetes Care, vol. 32, no. 6, pp. 1023-1025, 2009.

[14] M. R. Olthof, A. E. Van Dijk, C. F. Deacon, R. J. Heine, and R. M. Van Dam, "Acute effects of decaffeinated coffee and the major coffee components chlorogenic acid and trigonelline on incretin hormones," Nutrition and Metabolism, vol. 8, article 10, 2011.

[15] T. Bakuradze, R. Lang, T. Hofmann et al., "Antioxidant effectiveness of coffee extracts and selected constituents in cellfree systems and human colon cell lines," Molecular Nutrition and Food Research, vol. 54, no. 12, pp. 1734-1743, 2010.

[16] W. J. Yen, B. S. Wang, L. W. Chang, and P. D. Duh, "Antioxidant properties of roasted coffee residues," Journal of Agricultural and Food Chemistry, vol. 53, no. 7, pp. 2658-2663, 2005.

[17] J. Zhou, S. Zhou, and S. Zeng, "Experimental diabetes treated with trigonelline: effect on beta cell and pancreatic oxidative parameters," Fundamental \& Clinical Pharmacology. In press.

[18] J. Y. Zhou, S. W. Zhou, S. Y. Zeng, J. Y. Zhou, M. J. Jiang, and Y. He, "Hypoglycemic and hypolipidemic effects of ethanolic extract of mirabilis jalapa L. Root on normal and diabetic mice," Evidence-Based Complementary and Alternative Medicine, vol. 2012, Article ID 257374, 9 pages, 2012.

[19] A. Harkavyi and P. S. Whitton, "Glucagon-like peptide 1 receptor stimulation as a means of neuroprotection," British Journal of Pharmacology, vol. 159, no. 3, pp. 495-501, 2010.

[20] T. Perry, H. W. Holloway, A. Weerasuriya et al., "Evidence of GLP-1-mediated neuroprotection in an animal model of pyridoxine-induced peripheral sensory neuropathy," Experimental Neurology, vol. 203, no. 2, pp. 293-301, 2007.

[21] T. Himeno, H. Kamiya, K. Naruse et al., "Beneficial effects of exendin-4 on experimental polyneuropathy in diabetic mice," Diabetes, vol. 60, no. 9, pp. 2397-2406, 2011.

[22] R. Bianchi, I. Cervellini, C. Porretta-Serapiglia et al., "Beneficial effects of PKF275-055, a novel, selective, orally bioavailable, long-acting dipeptidyl peptidase IV inhibitor in streptozotocin-induced diabetic peripheral neuropathy," The Journal of Pharmacology and Experimental Therapeutics, vol. 340, no. 1, pp. 64-72, 2012.

[23] W. J. Liu, H. Y. Jin, K. A. Lee, S. H. Xie, H. S. Baek, and T. S. Park, "Neuroprotective effect of the glucagon-like peptide1 receptor agonist, synthetic exendin-4, in streptozotocininduced diabetic rats," British Journal of Pharmacology, vol. 164, no. 5, pp. 1410-1420, 2011.

[24] H. Y. Jin, W. J. Liu, J. H. Park, H. S. Baek, and T. S. Park, "Effect of Dipeptidyl Peptidase-IV (DPP-IV) Inhibitor (Vildagliptin) on Peripheral Nerves in Streptozotocin-induced Diabetic Rats," Archives of Medical Research, vol. 40, no. 7, pp. 536-544, 2009.

[25] Y. Li, T. Perry, M. S. Kindy et al., "GLP-1 receptor stimulation preserves primary cortical and dopaminergic neurons in cellular and rodent models of stroke and Parkinsonism," Proceedings of the National Academy of Sciences of the United States of America, vol. 106, no. 4, pp. 1285-1290, 2009.

[26] C. G. Jolivalt, M. Fineman, C. F. Deacon, R. D. Carr, and N. A. Calcutt, "GLP-1 signals via ERK in peripheral nerve and prevents nerve dysfunction in diabetic mice," Diabetes, Obesity \& Metabolism, vol. 13, no. 11, pp. 990-1000, 2011.

[27] S. Agthong and D. R. Tomlinson, "Inhibition of p38 MAP kinase corrects biochemical and neurological deficits in experimental diabetic neuropathy," Annals of the New York Academy of Sciences, vol. 973, pp. 359-362, 2002.

[28] S. X. Jin, Z. Y. Zhuang, C. J. Woolf, and R. R. Ji, "p38 mitogenactivated protein kinase is activated after a spinal nerve ligation in spinal cord microglia and dorsal root ganglion neurons and contributes to the generation of neuropathic pain," Journal of Neuroscience, vol. 23, no. 10, pp. 4017-4022, 2003. 
[29] J. Y. Zhou, S. W. Zhou, K. B. Zhang et al., "Chronic effects of berberine on blood, liver glucolipid metabolism and liver PPARs expression in diabetic hyperlipidemic rats," Biological and Pharmaceutical Bulletin, vol. 31, no. 6, pp. 1169-1176, 2008.

[30] C. Courteix, A. Eschalier, and J. Lavarenne, "Streptozocininduced diabetic rats: behavioural evidence for a model of chronic pain," Pain, vol. 53, no. 1, pp. 81-88, 1993.

[31] K. A. Sullivan, J. M. Hayes, T. D. Wiggin et al., "Mouse models of diabetic neuropathy," Neurobiology of Disease, vol. 28, no. 3, pp. 276-285, 2007.

[32] A. M. Vincent, J. W. Russell, K. A. Sullivan et al., "SOD2 protects neurons from injury in cell culture and animal models of diabetic neuropathy," Experimental Neurology, vol. 208, no. 2, pp. 216-227, 2007.

[33] L. J. Coppey, J. S. Gellett, E. P. Davidson, J. A. Dunlap, D. D. Lund, and M. A. Yorek, "Effect of antioxidant treatment of streptozotocin-induced diabetic rats on endoneurial blood flow, motor nerve conduction velocity, and vascular reactivity of epineurial arterioles of the sciatic nerve," Diabetes, vol. 50, no. 8, pp. 1927-1937, 2001.

[34] A. Guven, O. Yavuz, M. Cam, C. Comunoglu, and O. Sevinc, "Central nervous system complications of diabetes in streptozotocin-induced diabetic rats: a histopathological and immunohistochemical examination," International Journal of Neuroscience, vol. 119, no. 8, pp. 1155-1169, 2009.

[35] K. J. Livak and T. D. Schmittgen, "Analysis of relative gene expression data using real-time quantitative PCR and the 2$\Delta \Delta$ CT method," Methods, vol. 25, no. 4, pp. 402-408, 2001.

[36] M. H. Kedees, M. Grigoryan, Y. Guz, and G. Teitelman, "Differential expression of glucagon and glucagon-like peptide 1 receptors in mouse pancreatic alpha and beta cells in two models of alpha cell hyperplasia," Molecular and Cellular Endocrinology, vol. 311, no. 1-2, pp. 69-76, 2009.

[37] J. Zhou and S. Zhou, "Berberine regulates peroxisome proliferator-activated receptors and positive transcription elongation factor b expression in diabetic adipocytes," European Journal of Pharmacology, vol. 649, no. 1-3, pp. 390-397, 2010.

[38] P. Kakkar, B. Das, and P. N. Viswanathan, "A modified spectrophotometric assay of superoxide dismutase," Indian Journal of Biochemistry and Biophysics, vol. 21, no. 2, pp. 130$132,1984$.

[39] H. H. Draper and M. Hadley, "Malondialdehyde determination as index of lipid peroxidation," Methods in Enzymology, vol. 186, pp. 421-431, 1990.

[40] V. Carozzi, A. Chiorazzi, A. Canta et al., "Effect of the chronic combined administration of cisplatin and paclitaxel in a rat model of peripheral neurotoxicity," European Journal of Cancer, vol. 45, no. 4, pp. 656-665, 2009.

[41] A. M. Vincent, J. W. Russell, P. Low, and E. L. Feldman, "Oxidative stress in the pathogenesis of diabetic neuropathy," Endocrine Reviews, vol. 25, no. 4, pp. 612-628, 2004.

[42] R. Bianchi, B. Buyukakilli, M. Brines et al., "Erythropoietin both protects from and reverses experimental diabetic neuropathy," Proceedings of the National Academy of Sciences of the United States of America, vol. 101, no. 3, pp. 823-828, 2004.

[43] A. M. Vincent, L. M. Hinder, R. Pop-Busui, and E. L. Feldman, "Hyperlipidemia: a new therapeutic target for diabetic neuropathy," Journal of the Peripheral Nervous System, vol. 14, no. 4, pp. 257-267, 2009.

[44] F. A. White, H. Jung, and R. J. Miller, "Chemokines and the pathophysiology of neuropathic pain," Proceedings of the
National Academy of Sciences of the United States of America, vol. 104, no. 51, pp. 20151-20158, 2007.

[45] T. Purves, A. Middlemas, S. Agthong et al., "A role for mitogen-activated protein kinases in the etiology of diabetic neuropathy," FASEB Journal, vol. 15, no. 13, pp. 2508-2514, 2001.

[46] T. A. Temraz, W. E. Houssen, M. Jaspars et al., "A pyridinium derivative from Red Sea soft corals inhibited voltage-activated potassium conductances and increased excitability of rat cultured sensory neurones," BMC Pharmacology, vol. 6, no. 10, pp. 1-19, 2006.

[47] C. Tohda, N. Nakamura, K. Komatsu, and M. Hattori, "Trigonelline-induced neurite outgrowth in human neuroblastoma SK-N-SH cells," Biological and Pharmaceutical Bulletin, vol. 22, no. 7, pp. 679-682, 1999.

[48] C. Tohda, T. Kuboyama, and K. Komatsu, "Search for natural products related to regeneration of the neuronal network," NeuroSignals, vol. 14, no. 1-2, pp. 34-45, 2005.

[49] A. K. Saini, A. Kumar, and S. S. Sharma, "Preventive and curative effect of edaravone on nerve functions and oxidative stress in experimental diabetic neuropathy," European Journal of Pharmacology, vol. 568, no. 1-3, pp. 164-172, 2007.

[50] A. Kumar, R. K. Kaundal, S. Iyer, and S. S. Sharma, "Effects of resveratrol on nerve functions, oxidative stress and DNA fragmentation in experimental diabetic neuropathy," Life Sciences, vol. 80, no. 13, pp. 1236-1244, 2007.

[51] A. E. Valsecchi, S. Franchi, A. E. Panerai, P. Sacerdote, A. E. Trovato, and M. Colleoni, "Genistein, a natural phytoestrogen from soy, relieves neuropathic pain following chronic constriction sciatic nerve injury in mice: anti-inflammatory and antioxidant activity," Journal of Neurochemistry, vol. 107, no. 1, pp. 230-240, 2008.

[52] R. Stavniichuk, V. R. Drel, H. Shevalye et al., "Baicalein alleviates diabetic peripheral neuropathy through inhibition of oxidative-nitrosative stress and p38 MAPK activation," Experimental Neurology, vol. 230, no. 1, pp. 106-113, 2011. 


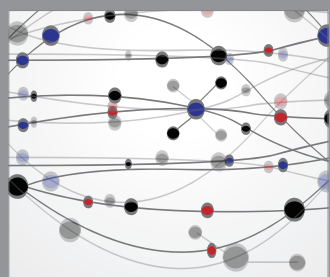

The Scientific World Journal
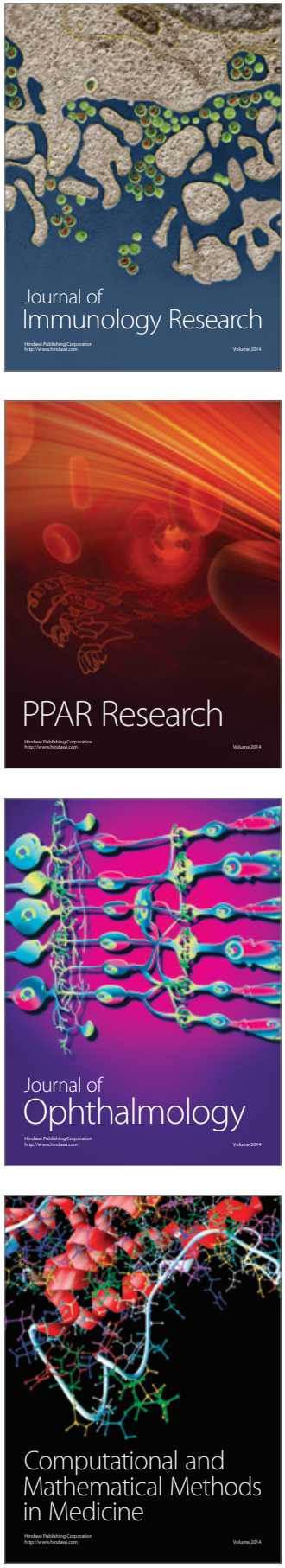

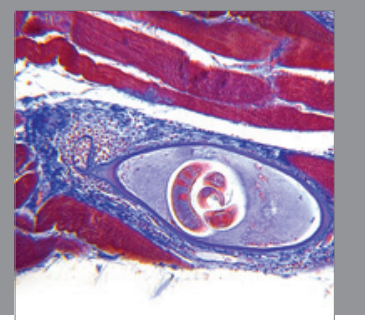

Gastroenterology

Research and Practice
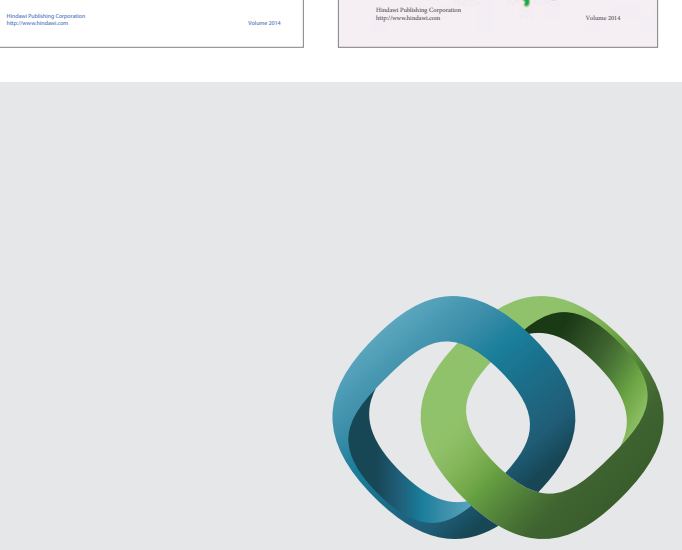

\section{Hindawi}

Submit your manuscripts at

http://www.hindawi.com
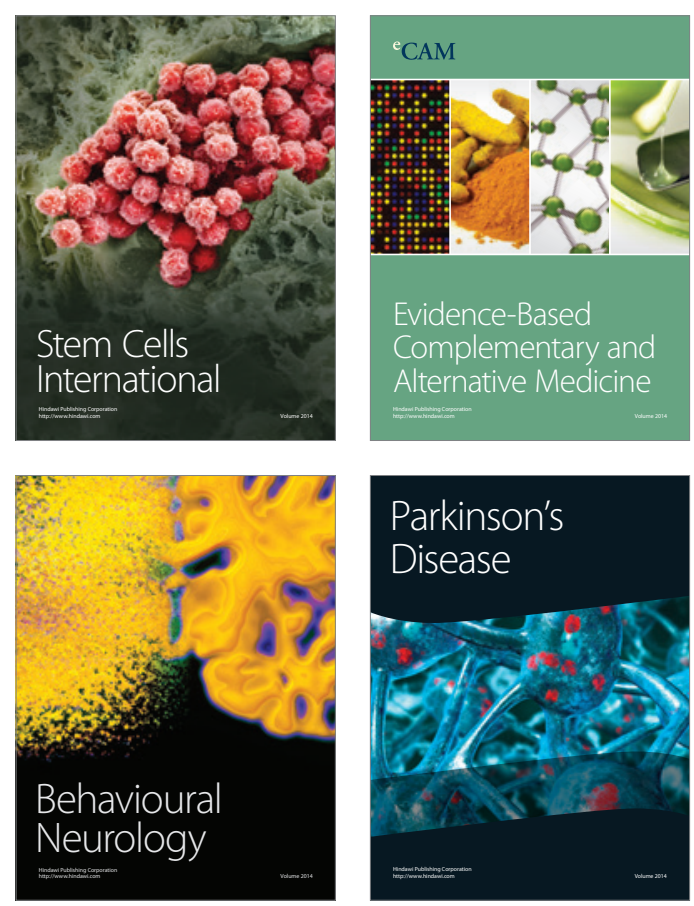

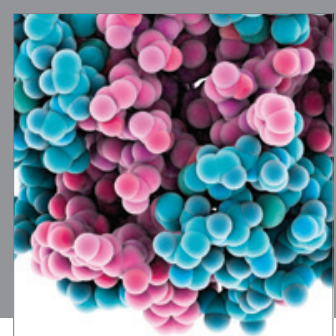

Journal of
Diabetes Research

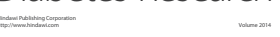

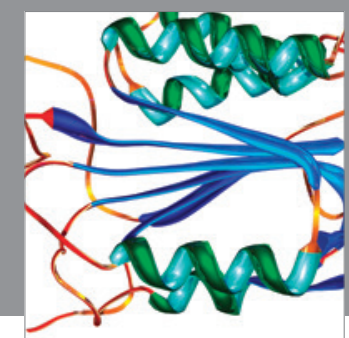

Disease Markers
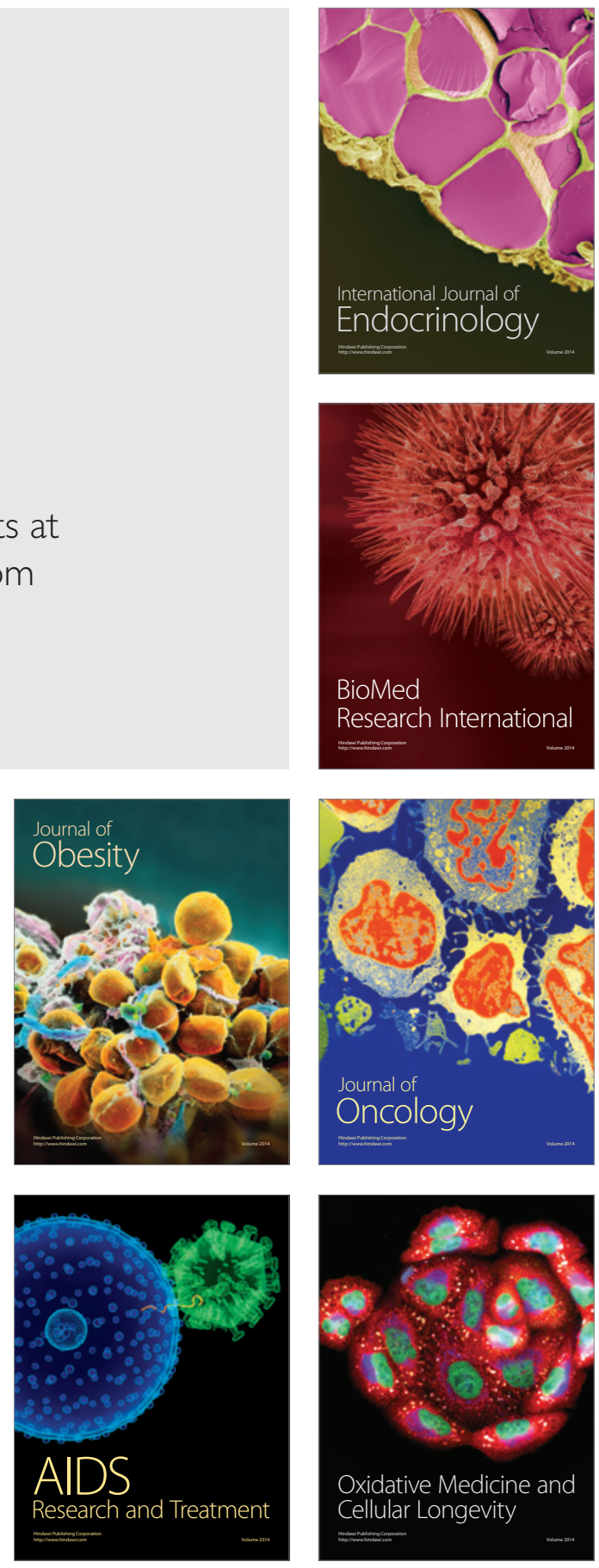